\title{
Stroke risk and NSAIDs: an Australian population-based study
}

Gillian E Caughey BSc(Hons), PhD, Research Fellow

Elizabeth E Roughead BPharm, MAppSc, PhD, ARC Future Fellow

Nicole Pratt

BSc, PhD Research Fellow

Graeme Killer AO, MB BS, MSC

Principal Medical Adviser ${ }^{2}$

Andrew L Gilbert BPharm, DipAppPsych, PhD, Professor

1 Quality Use of Medicines and Pharmacy Research Centre, Sansom Institute, University of South Australia, Adelaide, SA

2 Department of Veterans' Affairs, Canberra, ACT.

gillian.caughey@ unisa.edu.au

MJA 2011; 195: 525-529 doi: 10.5694/mjall.10055

Editorial p 488 yclooxygenase (COX)-2-selective non-steroidal anti-inflammatory drugs (NSAIDs) were developed to reduce the risk of gastrointestinal toxicity, an adverse event commonly associated with "traditional" non-selective NSAIDs. ${ }^{1}$ However, randomised clinical trials reported an increased risk of cardiovascular events and death for the COX-2-selective NSAID rofecoxib, leading to its worldwide withdrawal from the market. ${ }^{1-3}$ The results have been confirmed in several large-scale observational studies. ${ }^{4}$ Subsequent meta-analyses of randomised clinical trials and observational studies have included several traditional NSAIDs, and these have also been associated with an increased risk of cardiovascular events, ${ }^{4-6}$ with risk varying across individual non-selective and COX-2-selective NSAIDs. ${ }^{7,8}$ Most of the studies to date have focused on a combined cardiovascular end point, ${ }^{4-6}$ and few have examined the association between NSAID use and risk of stroke. ${ }^{9-13}$

NSAIDs inhibit the synthesis of the COX-1-derived platelet thromboxane, a potent prothrombotic agent and vasoconstrictor, as well as inhibiting the synthesis by endothelial cells of prostacyclin, a potent, predominantly COX-2-derived antithrombotic agent and vasodilator. ${ }^{8}$ The balance of activities of these mediators is essential to maintain vascular homeostasis. Selective inhibition of COX-2 inhibits synthesis of prostacyclin, with little or no effect on thromboxane, ${ }^{14}$ leading to a prothrombotic state and the potential for increased cardiovascular events. Further, inhibition of COX-2 within the kidney can lead to sodium and water retention and elevation of blood pressure..$^{15}$ A recent meta-analysis reported an increase in systolic blood pressure of about $3 \mathrm{mmHg}$ with use of COX-2 inhibitors. ${ }^{15}$ Clinically, even a small increase in blood pressure can

\begin{abstract}
Objective: To determine the risk of stroke associated with non-steroidal anti-inflammatory drug (NSAID) use.

Design, setting and participants: Retrospective cohort study of 162065 Australian veterans with incident dispensing of an NSAID between 1 January 2001 and 31 December 2008, using prescription event sequence symmetry analysis.

Main outcome measures: Hospitalisation for stroke, ischaemic stroke or haemorrhagic stroke.

Results: The absolute risk of stroke was low: 7.1/1000 people/year. Incident use of NSAIDs was associated with a 1.88 times increased risk $(95 \% \mathrm{Cl}, 1.70-2.08)$ of hospitalisation for stroke (ischaemic or haemorrhagic) following first ever dispensing of an NSAID. This equates to an increased absolute risk of 13.4 strokes/1000 people/year. Significant positive associations between starting an NSAID and having a hospitalisation for stroke were found for most NSAIDs, with adjusted sequence ratios ranging from 1.44 (95\% Cl, 1.16-1.80) for indomethacin to $1.80(95 \% \mathrm{Cl}, 1.59-2.04)$ for rofecoxib.

Conclusions: Incident use of NSAIDs was associated with an increased risk of stroke. Increased awareness of the potential for serious adverse cardiovascular events, together with individual assessment of cardiovascular risk, careful deliberation of the balance between risk and benefits and appropriate supervision, is required when initiating NSAID therapy.
\end{abstract}

have implications for increased cardiovascular risk, with reports of $67 \%$ increased risk of stroke with a similar increase in blood pressure. ${ }^{16}$

We undertook an analysis of the Department of Veterans' Affairs (DVA) administrative claims database to determine if the risk of stroke was evident in incident NSAID users in the Australian veteran population. Additionally, since the selectivity for COX-2 over COX-1 inhibition varies between individual NSAIDs across classes, ${ }^{17,18}$ we examined the risk of stroke by COX-2 selectivity.

\section{Methods}

\section{Data source}

Data were sourced from the DVA administrative claims database that contains details of all prescription medicines, and medical and allied health services and hospitalisations subsidised by the DVA for a treatment population of 300000 veterans, war widows and widowers. Over $70 \%$ of the population are aged 70 years or older, $54 \%$ are men, and $9.8 \%$ live in residential aged care and within a year are dispensed an average of 11 unique medicines. ${ }^{19}$ Medicines are coded according to the World Health Organization (WHO) anatomical and therapeutic chemical (ATC) classification ${ }^{20}$ and the Schedule of Pharmaceutical Benefits item codes. ${ }^{21}$ Hospitalisations are coded according to the WHO International classification of diseases, 10th revision, Australian modification (ICD-10-AM) ${ }^{22}$

\section{Study design}

Prescription event sequence symmetry analysis ${ }^{23,24}$ was undertaken to examine the association between hospitalisation for stroke (ICD-10-AM, I60-I64) and the prescription of NSAIDs (ATC, M01A) using data from 1 January 2001 to 31 December 2008. For meloxicam, the analysis was from 1 January 2002 to 31 December 2008, and for rofecoxib, from 1 January 2002 to 31 December 2004, reflecting product entry and withdrawal 
1 Risk of first stroke after and before initiation of non-steroidal anti-inflammatory drug (NSAID) use, by incident stroke type and NSAID*

\begin{tabular}{|c|c|c|c|c|c|}
\hline $\begin{array}{l}\text { Incident stroke, } \\
\text { by NSAID }\end{array}$ & $\begin{array}{l}\text { No. of } \\
\text { patients }\end{array}$ & $\begin{array}{c}\text { COX-1/COX-2 } \\
\text { ratio }\left(\mathrm{IC}_{50}\right)\end{array}$ & $\begin{array}{l}\text { Stroke in } 12 \text { months } \\
\text { after initiation of } \\
\text { NSAID use }\end{array}$ & $\begin{array}{l}\text { Stroke in } 12 \text { months } \\
\text { before initiation of } \\
\text { NSAID use }\end{array}$ & $\begin{array}{l}\text { Adjusted } \\
\text { sequence ratio } \\
(95 \% \mathrm{Cl})\end{array}$ \\
\hline \multicolumn{6}{|l|}{ All stroke } \\
\hline Any NSAID & 1821 & & 1245 & 576 & $1.88(1.70-2.08)$ \\
\hline \multicolumn{6}{|c|}{ Non-selective NSAID } \\
\hline Ibuprofen & 345 & 0.5 & 193 & 152 & $1.23(0.99-1.52)$ \\
\hline Naproxen & 209 & 0.7 & 130 & 79 & $1.52(1.15-2.01)$ \\
\hline Indomethacin & 333 & 1.9 & 203 & 130 & $1.44(1.16-1.80)$ \\
\hline Piroxicam & 114 & 14.1 & 80 & 34 & $2.04(1.36-3.04)$ \\
\hline Meloxicam & 908 & 18.0 & 593 & 315 & $1.71(1.49-1.96)$ \\
\hline Diclofenac & 545 & 29.0 & 358 & 187 & $1.75(1.47-2.09)$ \\
\hline \multicolumn{6}{|c|}{ COX-2-selective NSAID } \\
\hline Celecoxib & 1036 & 30.0 & 654 & 382 & $1.51(1.33-1.71)$ \\
\hline Rofecoxib & 1179 & 267.0 & 811 & 368 & $1.80(1.59-2.04)$ \\
\hline \multicolumn{6}{|l|}{ Ischaemic stroke } \\
\hline Any NSAID & 910 & & 627 & 283 & $1.90(1.65-2.18)$ \\
\hline \multicolumn{6}{|c|}{ Non-selective NSAID } \\
\hline Ibuprofen & 180 & & 92 & 88 & $1.03(0.77-1.39)$ \\
\hline Naproxen & 99 & & 62 & 37 & $1.51(1.00-2.26)$ \\
\hline Indomethacin & 191 & & 113 & 78 & $1.35(1.01-1.80)$ \\
\hline Piroxicam & 51 & & 34 & 17 & $1.74(0.97-3.11)$ \\
\hline Meloxicam & 439 & & 284 & 155 & $1.66(1.37-2.02)$ \\
\hline Diclofenac & 268 & & 175 & 93 & $1.72(1.34-2.21)$ \\
\hline \multicolumn{6}{|c|}{ COX-2-selective NSAID } \\
\hline Celecoxib & 500 & & 320 & 180 & $1.55(1.30-1.87)$ \\
\hline Rofecoxib & 567 & & 384 & 183 & $1.71(1.43-2.04)$ \\
\hline \multicolumn{6}{|c|}{ Haemorrhagic stroke } \\
\hline Any NSAID & 350 & & 250 & 100 & $2.19(1.74-2.77)$ \\
\hline \multicolumn{6}{|c|}{ Non-selective NSAID } \\
\hline Ibuprofen & 70 & & 41 & 29 & $1.35(0.84-2.17)$ \\
\hline Naproxen & 48 & & 34 & 14 & $2.17(1.16-4.03)$ \\
\hline Indomethacin & 57 & & 42 & 15 & $2.36(1.31-4.26)$ \\
\hline Piroxicam & 28 & & 22 & 6 & $2.97(1.21-7.33)$ \\
\hline Meloxicam & 210 & & 143 & 67 & $1.88(1.41-2.51)$ \\
\hline Diclofenac & 115 & & 78 & 37 & $1.92(1.30-2.85)$ \\
\hline \multicolumn{6}{|c|}{ COX-2-selective NSAID } \\
\hline Celecoxib & 193 & & 131 & 62 & $1.81(1.34-2.45)$ \\
\hline Rofecoxib & 216 & & 161 & 55 & $2.40(1.77-3.26)$ \\
\hline
\end{tabular}

COX $=$ cyclooxygenase. $*$ Classified by selectivity for COX-2 inhibition ${ }^{21,26}$ based on $\mathrm{IC}_{50}$ (half maximal inhibitory concentration) values. ${ }^{17,18}$ scribing or event trends over time. For example, if a medicine is prescribed with increasing incidence, a non-specific excess of patients with that medicine prescribed second would be expected. ${ }^{23}$ To adjust for such temporal trends, a null-effect sequence ratio is calculated, which estimates the sequence ratio one would expect in the absence of a causal association, given the incident medicine use and events in the background population. A description of the formula used for this calculation is provided elsewhere. ${ }^{23}$ An adjusted sequence ratio (ASR) is obtained by dividing the crude sequence ratio by the null-effect ratio and calculating 95\% confidence intervals. ${ }^{23,24}$ The ASR can then be interpreted as the incident rate ratio of stroke in NSAID exposed versus nonexposed person-time. ${ }^{23}$ Prescription event sequence symmetry analyses were restricted to sequences of incident NSAIDs and stroke hospitalisations within 12 months of each other, to limit the effect of age and other potential time-varying covariates on the probability of exposure and outcome.

The database was searched for all incident prescriptions of NSAIDs (ATC, M01A) (Box 1) and incident hospitalisations for stroke (ICD-10AM, I60-I64). For patients who had incident NSAID prescription and stroke hospitalisation within a 12month period, we ascertained the prescription event sequence, determined the sequence and adjusted sequence ratios, and calculated $95 \%$ confidence intervals.

A sensitivity analysis was also conducted to confirm the robustness of our primary findings. The analysis was limited to those patients who were incident users of an NSAID within a 12-month period - that is, those who had no previous dispensing of any NSAID for at least 12 months before incident NSAID dispensing. Additionally, patients were only included in the analysis if they did not switch NSAIDs in the 12 months after incident NSAID dispensing.

All analyses were performed using SAS version 9.1 (SAS Institute Inc, Cary, NC, USA). 


\section{Results}

A total of 162065 people with incident dispensing of an NSAID were identified in the study period. The average age was 76 years $(\mathrm{SD} \pm 7.9)$ and $60 \%$ were men. Of the individual NSAIDs, the four with the highest COX-2 selectivity were the most commonly initiated, ranging from 47141 incident dispensings for diclofenac to 79193 for celecoxib between 2001 and 2008 . There were 49489 incident dispensings for rofecoxib between 2002 and 2004, and 73294 for meloxicam between 2002 and 2008. For the remaining NSAIDs, the number of incident dispensings ranged from 10822 for piroxicam to 26143 for ibuprofen.

The absolute risk of stroke in our study was low: 7.1 strokes/1000 people/year, derived from those who had a stroke in the 12 months before incident NSAID use. However, analysis showed a 1.88 times (95\% CI, 1.702.08) increased risk of hospitalisation for stroke (ischaemic or haemorrhagic) following incident dispensing of an NSAID (Box 1), with a strong temporal association observed (Box 2). This equates to an increased absolute risk of 13.4 strokes/1000 people/ year. Significant positive associations between starting an NSAID and having a hospitalisation for stroke were found for all NSAIDs except ibuprofen, with ASRs ranging from 1.44 (95\% CI, 1.16-1.80) for indomethacin to 1.80 (95\% CI, 1.59-2.04) for rofecoxib (Box 1).

Examination by specific type of stroke showed ischaemic stroke to be the most prevalent, and incident use of an NSAID was associated with a 1.90 times increased risk $(95 \% \mathrm{CI}$, 1.65-2.18) of hospitalisation for ischaemic stroke (Box 1). Ibuprofen and piroxicam were not significantly associated with ischaemic stroke. Rofecoxib and diclofenac had the greatest increased risk for ischaemic stroke with ASRs of 1.71 (95\% CI, 1.43-2.04) and 1.72 (95\% CI, 1.342.21), respectively.

Incident use of any NSAID was associated with a more than doubled increased risk (ASR, 2.19; 95\% CI, 1.74-2.77) of haemorrhagic stroke (Box 1). Of the individual NSAIDs, only ibuprofen was not associated with haemorrhagic stroke. The increased risk of haemorrhagic stroke
2 Temporal association between initiation of NSAID use and incident stroke (ischaemic or haemorrhagic)

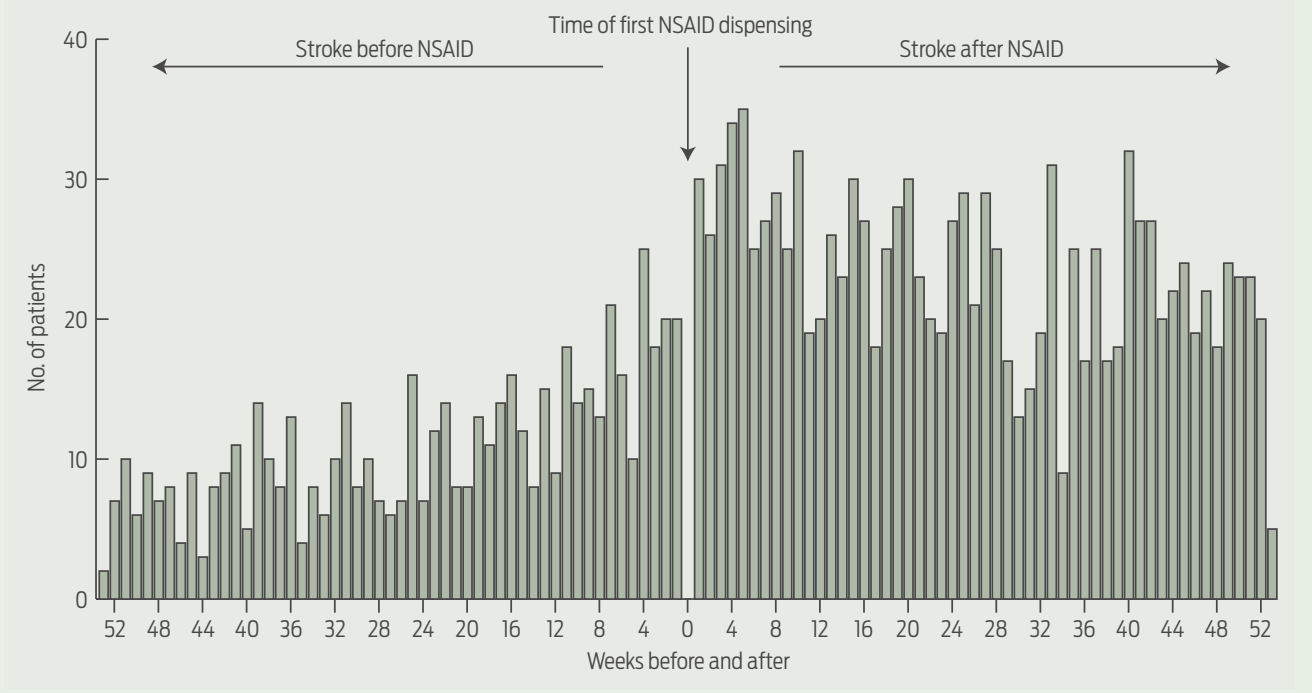

following incident NSAID use ranged from 1.81 times for celecoxib $(95 \% \mathrm{CI}$, $1.34-2.45)$ to 2.40 times for rofecoxib (95\% CI, 1.77-3.26). Piroxicam had high-risk estimates for haemorrhagic stroke (ASR, 2.97; 95\% CI, 1.21-7.33) (Box 1).

Sensitivity analyses (Box 3) suggest that our primary findings are robust. Initiation of any NSAID was associated with a 1.85 times increased risk of all stroke (95\% CI, 1.66-2.05) — an almost identical ASR to our primary finding. Some differences in sequence ratios between primary and sensitivity analyses were observed for individual NSAIDs. Sensitivity analysis found a significant positive association between ibuprofen and first stroke

(ASR, 1.74; 95\% CI, 1.15-2.65), and the increased risk of stroke for both naproxen and indomethacin was no longer statistically significant. For all other NSAIDs, except celecoxib, the risk estimate for increased risk of stroke increased (Box 3).

\section{Discussion}

The results from this Australian population-based study demonstrate that initiation of NSAIDs is associated with an increased risk of incident stroke, including ischaemic and haemorrhagic, in the older veteran population. However, the absolute risk of stroke is low and the increased risk is thus small. Nevertheless, small

\begin{tabular}{|c|c|c|c|c|}
\hline $\begin{array}{l}\text { All incident } \\
\text { stroke, by NSAID }\end{array}$ & $\begin{array}{l}\text { No. of } \\
\text { patients }\end{array}$ & $\begin{array}{c}\text { Stroke in } \\
12 \text { months } \\
\text { after initiation } \\
\text { of NSAID use }\end{array}$ & $\begin{array}{l}\text { Stroke in } \\
12 \text { months } \\
\text { before initiation } \\
\text { of NSAID use }\end{array}$ & $\begin{array}{l}\text { Adjusted sequence } \\
\text { ratio }(95 \% \mathrm{Cl})\end{array}$ \\
\hline Any NSAID & 1605 & 1094 & 511 & $1.85(1.66-2.05)$ \\
\hline \multicolumn{5}{|c|}{ Non-selective NSAID } \\
\hline Ibuprofen & 97 & 63 & 34 & $1.74(1.15-2.65)$ \\
\hline Naproxen & 52 & 31 & 21 & $1.39(0.80-2.40)$ \\
\hline Indomethacin & 79 & 49 & 30 & $1.53(0.97-2.41)$ \\
\hline Piroxicam & 28 & 22 & 6 & $3.13(1.27-7.72)$ \\
\hline Meloxicam & 181 & 111 & 70 & $1.51(1.12-2.03)$ \\
\hline Diclofenac & 149 & 103 & 46 & $1.96(1.39-2.78)$ \\
\hline \multicolumn{5}{|c|}{ COX-2-selective NSAID } \\
\hline Celecoxib & 490 & 329 & 161 & $1.71(1.42-2.07)$ \\
\hline Rofecoxib & 363 & 260 & 103 & $1.95(1.55-2.45)$ \\
\hline
\end{tabular}

* Limited to incident users of an NSAID within a 12-month period: ie, patients who had no previous dispensing of any NSAID for at least 12 months before incident NSAID dispensing and those who did not switch NSAIDs in the 12 months after incident NSAID dispensing. $\dagger$ Classified by selectivity for cyclooxygenase-2 inhibition ${ }^{21,26}$ based on IC $_{50}$ (half maximal inhibitory concentration) values. ${ }^{77,18}$ 
increases in risk may be particularly important for older people, who commonly have comorbidities that are associated with increased risk of stroke. At least a third of the older population will have cardiovascular disease (CVD) and 20\% will have comorbid arthritis and CVD, ${ }^{27}$ meaning that NSAIDs will commonly be used by patients who are at risk of future cerebrovascular events.

Our results are in accord with previous studies, further strengthening the evidence for the association between NSAID use and increased risk of stroke, with the more potent COX-2-selective NSAIDs such as rofecoxib generally associated with greatest risk of stroke. ${ }^{9-13}$ In a population-based study of over 7000 people in the Netherlands, use of any NSAID (including non-selective and COX-2selective NSAIDs) was associated with a $77 \%$ (95\% CI, 1.29-2.41) increased risk of stroke. ${ }^{9}$ Examination of individual NSAIDs in a large United States study of over 300000 people showed a 50\% (95\% CI, 1.081.98) increased risk of stroke in new users of the highly COX-2-selective NSAID rofecoxib. ${ }^{10}$ A more recent Danish study further supports these results, finding a dose-dependent increased risk of stroke (either fatal or non-fatal) associated with NSAID use in healthy individuals. ${ }^{11,12}$ The increased risk of stroke ranged from $28 \%$ with ibuprofen (95\% CI, 1.14 $1.44)$ to $86 \%$ with diclofenac $(95 \% \mathrm{CI}$, 1.58-2.19). Since the publication of the Danish study, there has been considerable media coverage within Australia about the safety of NSAIDs. ${ }^{28,29}$ Given the widespread use and easy access to NSAIDs in Australia, the Therapeutic Goods Administration announced that it was closely monitoring NSAID use. ${ }^{30}$

An increased risk of stroke was also observed in our study with the commonly used NSAID diclofenac, which has similar selectivity for COX-2 as celecoxib, but is available without a prescription. We observed that diclofenac was associated with a similar increase in risk of stroke (including ischaemic and haemorrhagic) to that of rofecoxib, which was withdrawn from the market in 2004 because of concerns about its cardiovascular safety. In general, the non- selective NSAID naproxen and, to a lesser degree, ibuprofen (particularly low-dose) have been reported as having better cardiovascular safety than other NSAIDs. ${ }^{10,11}$ These NSAIDs are similar in terms of their lack of selectivity for COX-2. However, in our study, the evidence for risk of stroke with these non-selective NSAIDs is less clear.

While the majority of studies to date have reported an increased risk of stroke with NSAID use, there are some conflicting results. ${ }^{5,31}$ One study in the veteran population reported no significant association between NSAID use and ischaemic stroke. ${ }^{31}$ However, its authors acknowledge that they did not differentiate between incident and prevalent NSAID users (the inclusion of prevalent users would reduce the effect of NSAID exposure towards the null, as a result of potential survivor bias).

Increased risk of cardiovascular events associated with NSAID use has been reported in high-risk patients with established CVD, ${ }^{6,32}$ highlighting the importance of considering individual patient characteristics when determining which NSAID should be prescribed. Current guidelines advocate the use of paracetamol as firstline therapy for pain management, and if an NSAID is deemed to be required, the lowest dose should be prescribed for the shortest duration. ${ }^{33}$ Non-NSAID analgesic strategies might also be considered for such patients.

The association of increasing COX2 selectivity with increasing risk of stroke is less evident in our study, and it is likely that a number of factors contribute to the overall cardiovascular safety of NSAIDs. Variations in the pharmacodynamics of different NSAIDs have been reported to be independent predictors of cardiovascular risk, with slow-release formulations posing a greater risk of myocardial infarction, presumably as a direct consequence of prolonged drug exposure. ${ }^{34}$ Increasing dose and length of exposure to NSAIDs have also been reported to raise the risk of stroke. ${ }^{11,13,34}$ Effects of NSAIDs on blood pressure, volume retention and other renal effects will further contribute to cardiovascular risk. ${ }^{15}$
We were unable to examine the use of over-the-counter, non-prescription NSAIDs. However, the veteran population has access to prescription medicines at a subsidised cost, and there is little incentive to purchase their medicine elsewhere. Our results are likely to be applicable to the Australian population. Age-specific comparisons between DVA gold card holders with no service-related disability and the wider Australian population have shown similar rates of general practitioner visits, use of prescriptions and hospitalisations. ${ }^{35}$

Prescription event sequence symmetry analysis provides a method for rapid assessment and uses the individual patients as their own controls, thereby minimising any potential bias caused by individual variations. ${ }^{23}$ Further, the sensitivity analysis included only incident users who did not switch NSAIDs, potentially reducing bias where developing the outcome can increase with time. However, the analysis is associative only and does not prove causality. This type of analysis has been proposed as an important tool for post-marketing surveillance and monitoring of adverse drug events which have relatively short latency, using populationbased data. ${ }^{23}$ Undertaking this same analysis using the national data would further strengthen the evidence of association.

In conclusion, we report that incident use of NSAIDs is associated with an increased risk of stroke. Although rofecoxib has been withdrawn from the market, meloxicam, diclofenac and celecoxib accounted for almost two-thirds of all NSAID dispensings in 2008 in Australia, ${ }^{26}$ and all were shown to be significantly associated with increased stroke risk. Individual assessment of cardiovascular risk, careful deliberation of the balance between risk and benefits and appropriate supervision is required when initiating NSAID therapy. Enhanced patient awareness of the potential for serious adverse cardiovascular events with all NSAIDs may also attenuate risk.

Acknowledgements: Elizabeth Roughead was supported by an Australian Research Council Future Fellowship. We thank the Australian Department of Veterans' Affairs for providing the data used in this study.

Competing interests: No relevant disclosures. 
Received 18 Jan 2011, accepted 14 Jul 2011.

1 Bombardier C, Laine L, Reicin A, et al. Comparison of upper gastrointestinal toxicity of rofecoxib and naproxen in patients with rheumatoid arthritis. VIGOR Study Group. N Engl J Med 2000; 343: 1520-1528.

2 Bresalier RS, Sandler RS, Quan H, et al. Cardiovascular events associated with rofecoxib in a colorectal adenoma chemoprevention trial. N Engl J Med 2005; 352: 1092-1102.

3 Solomon SD, McMurray JJ, Pfeffer MA, et al. Cardiovascular risk associated with celecoxib in a clinical trial for colorectal adenoma prevention. NEngl J Med 2005; 352: 1071-1080.

4 McGettigan P, Henry D. Cardiovascular risk and inhibition of cyclooxygenase: a systematic review of the observational studies of selective and nonselective inhibitors of cyclooxygenase 2 . JAMA 2006; 296: 1633-1644.

5 Kearney PM, Baigent C, Godwin J, et al. Do selective cyclo-oxygenase- 2 inhibitors and traditional non-steroidal anti-inflammatory drugs increase the risk of atherothrombosis? Meta-analysis of randomised trials. BMJ 2006 332: 1302-1308.

6 Trelle S, Reichenbach S, Wandel S, et al. Cardiovascular safety of non-steroidal antiinflammatory drugs: network meta-analysis. $B M$ 2011; 342; c7086.

7 Hippisley-Cox J, Coupland C. Risk of myocardial infarction in patients taking cyclo-oxygenase-2 inhibitors or conventional non-steroidal antiinflammatory drugs: population based nested case-control analysis. BMJ 2005; 330: 1366

8 Graham DJ, Campen D, Hui R, et al. Risk of acute myocardial infarction and sudden cardiac death in patients treated with cyclo-oxygenase 2 selective and non-selective non-steroidal antiinflammatory drugs: nested case-control study. Lancet 2005; 365: 475-481.

9 Haag MDM, Bos MJ, Hofman A, et al. Cyclooxygenase selectivity of nonsteroidal antiinflammatory drugs and risk of stroke. Arch Intern Med 2008; 168: 1219-1224

10 Roumie CL, Mitchel EF Jr, Kaltenbach L, et al. Nonaspirin NSAIDs, cyclooxygenase 2 inhibitors, and the risk for stroke. Stroke 2008; 39: 20372045.

11 Fosbol EL, Folke F, Jacobsen S, et al. Causespecific cardiovascular risk associated with nonsteroidal antiinflammatory drugs among healthy individuals. Circ Cardiovasc Qual Outcomes 2010: 3: 395-405.

12 Gislason GH. Use of non-steroidal antiinflammatory drugs (NSAIDs) is associated with increased risk of stroke among healthy people. European Society of Cardiology Congress; 2010 Aug 28-Sep 1; Stockholm, Sweden.

13 Chang $\mathrm{CH}$, Shau WY Kuo CW et al. Increased risk of stroke associated with nonsteroidal antiinflammatory drugs: a nationwide casecrossover study. Stroke 2010; 41: 1884-1890.

14 Caughey GE, Cleland LG, Penglis PS, et al. Roles of cyclooxygenase (COX)-1 and COX-2 in prostanoid production by human endothelial cells: selective up-regulation of prostacyclin synthesis by COX-2. J Immunol 2001; 167: 2831-2838.

15 Chan CC, Reid CM, Aw TJ, et al. Do COX-2 inhibitors raise blood pressure more than nonselective NSAIDs and placebo? An updated meta-analysis. J Hypertens 2009; 27: 2332-2341.

16 Collins R, Peto R, MacMahon S, et al. Blood pressure, stroke, and coronary heart disease. Part 2 , short-term reductions in blood pressure: overview of randomised drug trials in their epidemiological context. Lancet 1990; 335: 827838.

17 Patrono C, Patrignani P, García Rodríguez LA. Cyclooxygenase-selective inhibition of prostanoid formation: transducing biochemical selectivity into clinical read-outs. J Clin Invest 2001; 108: 7-13.

18 Warner TD, Giuliano F, Vojnovic I, et al. Nonsteroid drug selectivities for cyclo-oxygenase-1 rather than cyclo-oxygenase- 2 are associated with human gastrointestinal toxicity: a full in vitro analysis. Proc Natl Acad Sci USA 1999; 96: 7563-7568.

19 Australian Government Department of Veterans' Affairs. Treatment population statistics. Quarterly report - June 2010. Canberra: DVA 2010. http://www.dva.gov.au/aboutDVA/ Statistics/Documents/TpopJun10.pdf (accessed Jun 2011).

20 World Health Organization Collaborating Centre for Drug Statistics Methodology. Anatomical therapeutic chemical code classification index with defined daily doses. http://www.whocc.no/ atcddd/ (accessed Sep 2011).

21 Australian Government Department of Health and Ageing. Schedule of pharmaceutical benefits. PBS for health professionals. 2011. http://www.pbs.gov.au/ (accessed Jun 2011)

22 National Centre for Classification in Health. International statistical classification of diseases and related health problems, 10th revision, Australian modification (ICD-10-AM). 7th ed. Sydney: NCCH, 2010.

23 Hallas J. Evidence of depression provoked by cardiovascular medication: a prescription sequence symmetry analysis. Epidemiology 1996; 7: 478-484.

24 Caughey GE, Roughead EE, Pratt N, et al. Increased risk of hip fracture in the elderly associated with prochlorperazine: is a prescribing cascade contributing? Pharmacoepidemiol Drug Saf 2010; 19: 977-982.

25 Langton PE, Hankey GJ, Eikelboom JW. Cardiovascular safety of rofecoxib (Vioxx): lessons learned and unanswered questions: we need processes in place to follow up suspicions about serious adverse events. Med J Aust 2004; 181: 524-525.

26 Australian Government Department of Health and Ageing. Australian statistics on medicine 2008. Canberra: Commonwealth of Australia, 2009.

27 Caughey GE, Ramsay EN, Vitry Al, et al. Comorbid chronic diseases, discordant impact on mortality in the elderly; a 14 year longitudinal population study. J Epidemiol Community Health 2010; 64: 1036-1042.

28 Corderoy A. Study links Voltaren and Nurofen to strokes. Sydney Morning Herald 2010; 14 Sep.

29 Corderoy A. Painkiller linked to stroke. The Age (Melbourne) 2010; 14 Sep.

30 Rose D. TGA monitoring drugs with stroke risk. MedConnect 2010; 14 Sep.

31 Mangoni AA, Woodman RJ, Gaganis P, et al. Use of non-steroidal anti-inflammatory drugs and risk of incident myocardial infarction and heart failure, and all-cause mortality in the Australian veteran community. Br J Clin Pharmacol 2010; 69: $689-700$.

32 Ray WA, Varas-Lorenzo C, Chung CP, et al. Cardiovascular risks of nonsteroidal antiinflammatory drugs in patients after hospitalization for serious coronary heart disease. Circ Cardiovasc Oual Outcomes 2009; 2: 155-163.

33 Solomon DH, Avorn J, Stürmer T, et al. Cardiovascular outcomes in new users of coxibs and nonsteroidal antiinflammatory drugs: highrisk subgroups and time course of risk. Arthritis Rheumatism 2006; 54: 1378-1389.

34 García Rodríguez LA, Tacconelli S, Patrignani P. Role of dose potency in the prediction of risk of myocardial infarction associated with nonsteroidal anti-inflammatory drugs in the general population. J Am Coll Cardiol 2008; 52: 1628-1636.

35 Australian Institute of Health and Welfare. Health care usage and costs. A comparison of veterans and war widows and widowers with the rest of the community. Canberra: AlHW, 2002. (AlHW Cat. No. PHE 42.) 